\title{
Trois textes en série : la consolation de Plutarque traduite par La Boétie - Montaigne - Céline Jean Vignes (éd.)
}

\section{(2) OpenEdition Journals}

\section{Édition électronique}

URL : http://journals.openedition.org/rhetorique/547

DOI : $10.4000 /$ rhetorique.547

ISSN : 2270-6909

Éditeur

UGA Éditions/Université Grenoble Alpes

\section{Édition imprimée}

ISBN : 978-2-37747-010-5

Référence électronique

"Trois textes en série : la consolation de Plutarque traduite par La Boétie - Montaigne - Céline ",

Exercices de rhétorique [En ligne], 9 | 2017, mis en ligne le 20 juin 2017, consulté le 12 septembre 2020. URL : http://journals.openedition.org/rhetorique/547 ; DOI : https://doi.org/10.4000/rhetorique.547

Ce document a été généré automatiquement le 12 septembre 2020.

\section{c) (i) (2) (2)}

Les contenus de la revue Exercices de rhétorique sont mis à disposition selon les termes de la Licence Creative Commons Attribution - Pas d'Utilisation Commerciale - Partage dans les Mêmes Conditions 4.0 International. 


\section{Trois textes en série : la consolation de Plutarque traduite par La Boétie - Montaigne - Céline \\ Jean Vignes (éd.)}

\section{La consolation de Plutarque à sa femme traduite par La Boétie (1571)}

Des cinq lettres de consolation composées par Plutarque, seules deux nous sont parvenues. Celle qu'il adresse à son épouse Timoxéna concerne la mort de leur fille du même nom, décédée à l'âge de deux ans à une date inconnue (probablement autour de 100 ap. J.-C.). La plus ancienne traduction française connue et la première imprimée (par les soins de Montaigne) est l'œuvre d'Étienne de La Boétie.

Édition de référence : La Mesnagerie de Xenophon. Les Regles de Mariage, de Plutarque. Lettre de consolation de Plutarque à sa femme. Le tout traduict de Grec en françois par feu M. Estienne de La Boétie, Conseiller du Roy en sa Cour de Parlement de Bordeaux, item un Discours sur la mort dudit Seigneur De la Boëtie par M. de Montaigne, Paris, Federic Morel, 1571, f. 90-99.

Édition moderne dans CEuvres complètes d'Estienne de La Boétie, éd. Paul Bonnefon, Genève, Slatkine Reprints, 1967, p 187-199.

Nous reproduisons le texte d'après l'édition originale, en dissimilant selon l'usage moderne $i$ et $j$, $u$ et $v$, en remplaçant l'esperluette \& par et et en modernisant souvent la ponctuation. Les accents sont ceux de l'originale (y compris élevez à 90 $\mathrm{v}^{\circ}$, és et aysément à $98 \mathrm{r}^{\circ}$ ); nous remplaçons partout par là les la de l'originale, dans 
ceux la, etc., l'originale n'étant pas toujours régulière sur ce point (à $95 \mathrm{v}^{\circ}$, il y a ce temps la et quelques lignes plus loin ce temps là).

La traduction de La Boétie n'est pas subdivisée en paragraphes ; nous adoptons la subdivision et la numérotation des paragraphes pratiquées dans les éditions et traductions modernes de Plutarque.

\section{LETTRE DE CONSOLATION DE PLUTARQUE À SA FEMME. Plutarque à sa femme, bonne et heureuse vie.}

1 [1] L'homme que tu m'envoyas pour me porter les nouvelles du trespas de l'enfant, se fourvoya, à mon avis, sur chemin en venant à Athenes, mais je l'entendy à Tanagre ${ }^{1}$, quand j'y fus arrivé. Quant à l'enterrement, je croy que tout est desja fait. De ma part, je desire que ce qui en a esté fait, soit en la sorte qu'il pourra estre mieux pour te donner à ceste heure, et à l'avenir moins d'occasion de fascherie. Mais si en cela tu as laissé de faire quelque chose dont tu eusses envie, et attens sur ce mon advis, fais la hardiment, si tu penses, cela estant fait, en estre plus à $\left[90 \mathrm{v}^{\circ}\right]$ ton aise. Mais ce sera mettant à part toute superfluité, et vaine superstition ${ }^{2}$ : aussi sçay-je bien que de ces passions là, tu n'en tiens rien.

2 [2] D'une chose sans plus te veux-je avertir, qu'en ceste douleur tu te maintiennes, et à toy et à moy, dans les termes du devoir. Car de mon costé, je cognois et compren en cest inconvenient ${ }^{3}$, de combien il est grand. Mais si je trouve à mon arrivée que tu te tormentes outre mesure, cela certes me troublera encores plus que l'accident mesme. Et pour vray, je ne suis ny de bois, ny de pierre : Toy-mesmes, le scais bien, m'ayant tousjours tenu compaignie à nourrir ${ }^{4}$ en commun tant d'enfants que nous avons eu, qui ont esté tous élevez et entretenus chez nous par nous mesmes. Et si sçais ${ }^{5}$ bien qu'apres avoir eu quatre enfans masles, toy ayant grande envie d'avoir une fille, ceste icy nasquit, et me donna occasion de luy mettre le mesme nom que tu portes ${ }^{6}$, aymé de moy uniquement ${ }^{7}$. Et vois bien encore qu'en notre mutuel amour il y a, outre ces occasions, quelque particuliere pointe d'une vive affection, en raison de la façon gaye qu'elle avoit ${ }^{8}$, et du tout ${ }^{9}$ franche et naïve, [91 $\mathrm{r}^{\circ}$ ] n'ayant rien de cholere et de despit : Et voyoit on en elle une nature admirable, paisible, douce, et attrempée ${ }^{10}$. Et l'amour qu'elle rendoit à ceux qui l'aymoient, et la recognoissance qu'elle avoit envers ceux qui luy faisoient quelque bien, donnoit tout à la fois plaisir, et cognoissance d'un naturel humain et debonnaire ${ }^{11}$.

[3] Or ma femme je ne sçay pas pourquoy toutes ces façons, qui elle vivant nous donnoient tant de plaisir, maintenant nous donneront peine, et nous travailleront ${ }^{12}$ quand nous y penserons : mais aussi je crains qu'en voulant chasser la douleur, nous ne chassons tout d'un coup la souvenance ${ }^{13}$, comme faisoit Climene qui dit :

Je me desplais des lieux où la jeunesse

$\left[91 \mathrm{v}^{\circ}\right]$ A escrimer, et à luitter s'addresse ${ }^{14}$,

Les arcs aysez de cormier me desplaisent ${ }^{15}$ :

tant elle craignoit et fuyoit le souvenir de son fils qui tousjours l'accompagnoit : car nostre nature fuit volontiers cela dont elle reçoit peine. Or faut-il, tout ainsi comme elle se rendoit telle en nostre endroit, qu'elle nous faisoit sentir tous les plaisirs du monde à nous festoyer, et à se faire voir, à se faire ouyr ${ }^{16}$, que pareillement à ceste heure la 
souvenance d'elle demeure tousjours, et vive dedans nous, apportant avec soy un plaisir plus grand, mais de beaucoup, que non pas l'ennuy ${ }^{17}$ : Au moins si nous pensons, qu'il est raisonnable que nous mesmes nous tirions quelque proffit au besoing, es advertissemens que nous avons fait souvent à plusieurs autres. Il faut ${ }^{18}$ donc entretenir ceste plaisante memoire, et non pas mener deuil, et se desconforter ${ }^{19}$ tant et lamenter, qu'il semble à voir que pour l'ayse qu'on a receu quelquefois, on vueille maintenant rendre en payement au double de fascheries et d'ennuys ${ }^{20}$.

4 [4] Ceux qui viennent de là où tu es vers moy, m'ont bien rapporté une chose, pour raison de laquelle ils t'admirent grandement. [92 $\mathrm{r}^{\circ}$ ] C'est que tu n'as point pris nouvel habillement, n'en ${ }^{21}$ rien difformé ne gasté ta façon accoustumee, en toy, ny en tes chambrieres : qu'il n'y avoit à l'enterrement aucun appareil somptueux qui sentist sa pompe et superfluité : mais que le tout a esté conduit avec grande discretion, et sans bruit, à la compagnie seulement de noz plus proches parents. Quant est de moy ${ }^{22}$, sçachant bien, que tu ne prins ${ }^{23}$ jamais de belle robe pour aller aux jeux et à la feste, mais as tousjours pensé que la somptuosité ne sert de rien $^{24}$, non pas mesme pour la volupté, je ne me suis point esbahy ${ }^{25}$ d'entendre que tu ayes entretenu en la tristesse ton assurance et modeste simplicité. Aussi il ne faut pas seulement qu'une femme de bien se garde pure aux festins et aux jeux, mais qu'elle pense que l'ebranlement que fait en nous la douleur, et le mouvement des fascheries n'a pas moins besoing d'une ferme discretion qui combate, non pas comme plusieurs estiment, contre l'amour que naturellement nous devons aux nostres, mais contre les desordonnees passions de l'esprit. Car nous ottroyons cela à la naturelle affec[92 $\left.\mathrm{v}^{\circ}\right]$ tion d'honorer ceux qui sont morts, de les regretter, de s'en souvenir ${ }^{26}$. Mais certes ce desir desordonné de mener deuil, devoyant l'esprit, et le menant parmy les lamentations et travaux ${ }^{27}$, n'est pas moins vicieux à le bien prendre, que la dissolution aux voluptez. Mais on pardonne à la tristesse plus volontiers et à bon droit, d'autant que ce qui est en elle de vicieux, en lieu de plaisir, a tousjours avec soy la peine et l'amertume. Car qu'est ce qu'on pourroit trouver de plus deraisonnable, que de deffendre le rire excessif et la joie desordonnee, et puis apres se laisser $\mathrm{du}_{\mathrm{tout}}{ }^{28}$ aller aux debordements des pleurs et des plaintes qui viennent de la mesme source ${ }^{29}$ d'où part le plaisir desmesuré ? et quelle raison peuvent avoir plusieurs marys d'entrer en querelles avec leurs femmes pour les garder ${ }^{30} \mathrm{de}$ porter des senteurs et de l'ecarlate: si après ils leur accordent de se tondre pour le deuil, de prendre nouvelle teinture de robe noire, de demeurer en un lieu assises ou mal couchees sans se bouger ${ }^{31}$ ? choses certainement toutes indignes et mal avenables ${ }^{32}$. $\mathrm{Et}$, ce que je trouve encores plus nouveau, n'est ce pas chose estrange [93 $\left.\mathrm{r}^{\circ}\right]$ de voir qu'alors que les femmes frappent et tourmentent les valets et chambrieres outre mesure et sans raison, les marys empeschent cela et les en gardent: et quand elles mesmes sont vivement tourmentees et cruellement par elles mesmes, ils n'en font aucun compte ${ }^{33}$, et les laissent à ce besoing, et en ce trouble d'esprit, où elles auroient besoing de la bonté et douceur d'eux et de leur courtoisie?

5 [5] Mais entre nous, ô ma femme, nous n'avons jamais encores eu debat aucun pour ces choses : si n'aurons nous pas à ceste heure pour ceste icy, à mon advis. Car il n'y a un seul des philosophes qui nous ont hantez ${ }^{34}$ et cogneus, qui ne se soit esmerveillé de l'honneste humilité de ton accoustrement ${ }^{35}$, et de la moderation et façon reformée de ta $v^{1 e^{36}}$ : et n'y a pas un de noz citoyens, à qui ton honneste simplesse ${ }^{37}$ aux eglises ${ }^{38}$, aux sacrifices, aux theatres, ne serve d'exemple et de miroir. Et d'autrefois à un pareil besoing qu'à ceste heure, tu fis cognoistre une grande asseurance d'esprit, et fermeté 
de cueur, lorsque tu perdis l'aisné de noz enfants ${ }^{39}$, et encores depuis alors que nostre beau Charon nous abandonna. Car il me souvient [93 $\mathrm{v}^{\circ}$ ] qu'on me porta les nouvelles de la mort de ce garçon, ainsi que je descendois de sur mer, et lors plusieurs de mes hostes et amis me tindrent compagnie, et vindrent avecques moy en nostre maison, et beaucoup d'autres quand et eux ${ }^{40}$. Et puis voyant chez nous, que toutes choses estoient en leur ranc ${ }^{41}$, et tout paisible comme de coustume, ils pensoient (et ainsi l'ont ils dit depuis à maint-un ${ }^{42}$ ) qu'il n'y estoit rien avenu de mal, mais que quelqu'un avoit semé ceste fausse nouvelle; tant tu avois bien ordonné la maison en temps si triste, et qui donnoit si grande occasion de desordre. Et si avois tu nourry celuy la de tes propres mammelles, et pour luy avois enduré l'incision d'un tetin ${ }^{43}$ qui s'estoit fendu tout autour. Ce sont vrayement les chefs d'œuvre d'un bon cœur et noble, et d'une vive affection.

[6] Mais la pluspart des meres nous les voyons prenants entre leurs bras leurs enfants tant qu'ils vivent des mains d'autruy, pour les servir, ce semble, de passe-temps ${ }^{44}$ : et puis quand ils sont morts, indiscrettement ${ }^{45}$ elles s'abandonnent à un deuil vain et sans raison, non pas d'amitié, qu'elles ayent : car l'amitié certes est une belle chose et [94 $\mathrm{r}^{\circ}$ ] pleine de moderation et prevoyance : mais pour vray l'abondance d'une vaine ambition, qui est meslee avec un peu de passion naturelle, fait ce deuil ainsi sauvage et enragé, et ce grand desconfort ${ }^{46}$. Et qu'il soit ainsi ${ }^{47}$, il semble bien qu'Æsope ne l'ait pas ignoréé ${ }^{48}$ : car il dit, qu'alors que Jupiter partageoit les honneurs entre les Dieux, le deuil demanda sa part, et il luy en donna, mais seulement à l'endroit de ceux là qui de leur gré mesme luy en voudroyent faire. Ainsi doncques en avient il au commencement, car chacun deuil le meine luy mesme chez soy. Mais après quand il y a une fois gaigné place avec le temps vivant et logeant avec celuy qui l'a receu, il ne s'en va pas encores lorsqu'on luy donne congé. Donc il le faut combattre des l'entree à la porte, et non pas luy quitter le fort ${ }^{49}$, en laissant son habillement, et son poil ${ }^{50}$, et par tous autres pareils moyens, et toutes autres façons qui se presentans à toute heure devant les yeux, et attristant la personne, tiennent en serre ${ }^{51}$ et diminuent la vigueur de l'esprit, et le mettent en desespoir de trouver issue du mal, et le rendent incapable de consolation, tout obscur $\left[94 \mathrm{v}^{\circ}\right]$ et tenebreux, de tant que l'entendement depuis qu'il s'est par la douleur entourné ${ }^{52}$ et enveloppé de ces tristes habits, il ne se fait aucune part ny du rire en compagnie, ny de la lumiere, ny de la bonne chere, et de la plaisante et joyeuse table de ses amis. A ce mal de la tristesse se joint volontiers la nonchallance de sa personne et un despit contre la coutumiere façon jusques à ne se vouloir [ny frotter ${ }^{53}$ ny estuver ${ }^{54}$ : là où il failloit ${ }^{55}$ que l'esprit fist tout au contraire pour se secourir et ayder, par le moyen du corps sain et vigoureux: car certes quand le corps est sain et en sa gaillardise, une grand' partie de l'ennuy ${ }^{56}$ s'abbat et se relasche, comme le flot à un beau jour quand le temps calme : mais si on laisse le corps rouiller et durcir par le mal gouverner, et qu'il n'envoye plus rien de bon ny de gracieux à l'ame, ains ${ }^{57}$ seulement comme des ameres et fascheuses fumees: certes à grand' peine se peult-on ravoir, encores qu'on le vueille ${ }^{58}$. Si grandes sont les passions qui saisissent l'ame ainsi malmenee.

[7] Encore m'asseure-je tant de toy, que, pour ton regard en ceci, je ne crains point une chose, qui est bien en tel cas la plus grande et la plus à [95 $\left.\mathrm{r}^{\circ}\right]$ craindre : ce sont les visites d'un tas de mauvaises femmes ${ }^{59}$, leurs voix plaintives, et la recharge de leurs complaintes, avec lesquelles elles ont accoustumé de frotter, par maniere de dire, et refraischir et irriter la douleur, ne permettant point que le deuil, ou par autre moyen, ou bien de luy mesme, se vienne à flestrir, et s'amortisse. Car je sçay combien tu eus de 
peine n'a guieres, quand tu secourus si bien la sœur de Theon $^{60}$, et combatis si bien celles là, qui, outre le deuil qu'elle avoit en elle, luy faisoient encores, avec les assaults qu'elles luy donnoient, des cris et des pleurs, comme pour vray si elles eussent eu envie d'y mettre le feu. Car ${ }^{61}$ si on voit bruler la maison de son amy, on esteint la flamme le plus tost que l'on peut, et à la plus grande haste; et quand on le voit luy mesme se consommer en son esprit et tout enflammé, on luy attise encores le feu! Et certes on n'endure pas, quand quelqu'un a mal aux yeux, qu'il y mette la main, encores qu'il le vueille; et personne ne touche là où son mal luy cuit : et celuy qui est en deuil demeure tousjours assis, se presentant à tous venans expressement, ce semble, pour se faire esmou[95 $\left.\mathrm{v}^{\circ}\right]$ voir, et envenimer la playe, à fin que pour un peu de douleur qu'il a, qui le poingt et luy demange, l'ulcere egratigné s'empire tousjours, et devienne plus grand et fascheux. Or doncques de cela je suis certain que tu te garderas fort bien :

$8[8]^{62}$ mais encores essayes toy en ta pensee de te transferer toy-mesme et remettre à ce temps là, que ceste fille, maintenant morte, n'estoit pas encores née; et si ne pensions nous pas lors avoir occasion aucune de nous plaindre de la fortune. Puis, ayant pensé à ce temps là, assemble le avec celuy de maintenant, et tu trouveras que l'estat de nos affaires estoit lors, et est à celle heure entièrement pareil. Car, si nous estimons que nous avions plus de raison de nous contenter, avant qu'elle nasquit, il semblera, ma femme, que nous soyons courroucez et desplaisans de sa naissance. Or, les deux ans d'entre deux, qui a esté le terme de sa vie, il ne faut point les tirer hors ny rabbattre de nostre memoire ; mais, comme nous ayant apporté jouissance d'autant de faveur et de bien, les compter pour plaisir, et non pas reputer un bien court à grand mal, ny estre ingrats envers nostre fortune du present qu'elle $\left[96 \mathrm{r}^{\circ}\right]$ nous a fait, pour ce qu'elle ne l'a pas augmenté de tant comme nous esperions.

[9] ${ }^{63}$ Car certainement on ne peut faillir à tirer un bel et plaisant fruict de dire tousjours bien et se contenter de ce que Dieu a voulu, et de prendre à gré, et sans se plaindre, ce que la fortune nous baille. Et, en telles choses, celuy qui rameine le plus à soy la souvenance des biens passez, et qui destourne et retire l'entendement des choses qui le troublent et obscurcissent, pour le remettre en la partie de sa vie qu'il a trouvee la plus belle et la plus claire, c'est vrayement celuy la qui en esteint entierement sa douleur, ou, pour le moins, l'affoiblit et l'amortit, la destrempant avecques la meslange de son contraire. Car tout ainsi que ${ }^{64}$ les onguents de bonne odeur resjouissent tousjours le sentiment $t^{65}$, et si font un preservatif contre les mauvaises senteurs : ainsi le pensement du bien receu sert encore de remede necessaire au mal qui survient, au moins à celuy qui ne fuit pas la memoire du bien passé, et ne prent pas plaisir d'accuser entierement de tout la fortune: de quoy nous nous devons bien garder, et de vouloir calomnieusement blasmer la vie $\left[96 \mathrm{v}^{\circ}\right]$ d'entre nous hommes pour quelque tache de malheur, une possible sans plus ${ }^{66}$, qui se trouve en elle, comme en un livre, tout le demeurant estant net et entier. Car il te souvient bien de m'avoir souvent ouy dire que les changemens de fortune ne peuvent de guieres esbranler nostre vie, ny avec ses hazards elle ne luy sçauroit faire prendre grand saut ${ }^{67}$. Mais toute la felicité ne depend que d'une bonne et droite resolution, parfaite et accomplie en une habitude ferme et asseuree ${ }^{68}$. Et encores s'il faut, à la façon de la plus part des hommes, se gouverner par ce qui est hors de nous, et s'il est besoin de conter ce que nous tenons de la fortune, et faire le peuple mesme juge de nostre bon heur, ne prens pas garde, je te prie, aux larmes et plaintes de ceux qui te visitent maintenant, lesquels par une mauvaise coustume on voit faire ainsi, ains combien ceux là mesmes admirent ton bon heur, à raison des enfants que tu as, et de la grandeur de nostre maison, et de ta vie. Et, sans 
doute, ce seroit une chose merveilleusement desraisonnable qu'il n'y aye celuy de ceux qui te voyent, qui ne print volontiers la condition en [97 $\mathrm{r}^{\circ}$ ] quoy tu es, encores avec la charge de l'inconvenient dont toy et moy nous deuillons, et que tu fusses seule à t'en plaindre et mescontenter. Et n'y a pas de raison que le mal mesme qui te pique ne te face sentir combien nous devons à la fortune pour ce qu'il nous demeure. Certes, ny plus ny moins qu'on a veu quelques uns qui se sont amusez à tirer les vers d'Homere où il y a quelque faute au commencement ou à la fin, laissant ce pendant passer sans y prendre garde tant de belles et grandes inventions, ainsi seroit il de toy, si tu voulois rechercher curieusement les infortunes de ceste vie humaine, et, pour le regard des biens qui te viennent à foison et à monceaux, tomber en la mesme maladie des avares et riches mecaniques ${ }^{69}$, qui, ayans amassé de l'argent de toutes parts, n'en usent point, [mais se plaignent et se lamentent ${ }^{70}$ ] quand ils l'ont perdu. Or, si tu plains ta fille pour estre morte sans avoir esté mariee et porter enfants, tu as de l'autre costé de quoy te resjouir de ce qu'il n'y a aucun de ces biens là qui te defaillent ${ }^{71}$, et dont tu ne sois participante : car ce seroit bien folie de penser que ces biens fussent grands, au regard de ceux [97 $\mathrm{v}^{\circ}$ ] qui les ont perdus, et petits en l'endroit de ceux qui en jouissent. Davantage, si elle est allee en lieu où elle ne sente point la douleur, elle n'a pas besoin qu'on se deuille pour elle $\mathrm{e}^{72}$ : car pour quoy devons nous avoir mal pour raison d'elle, s'il n'y a rien qui luy en face ? Pour vray, la perte des grands biens doit faire cesser le deuil qu'on en pourroit mener, quand par la perte mesme on vient à cela de n'avoir plus besoing des choses perdues. Or, ta petite Timoxene n'a perdu que peu de bien, de tant qu'elle n'en cognoissoit que bien peu et se rejouissoit de peu. Car comment pourroit on dire qu'elle eust perdu ce dont elle n'avoit pas sentiment et qu'elle ne pouvoit encores ny cognoistre ny comprendre?

[10] Toutefois je sçay bien, touchant ceste opinion, qu'aucuns tiennent ${ }^{73}$ et la donnent à entendre à plusieurs, que les hommes, depuis qu'ils sont une fois dissous par la mort, n'ont en nul endroit nul mal ny tourment, je sçay bien, dis-je, quant à ceste opinion, que la religion de nostre païs te gardera de la croire, et les sentences qui se disent par mystere aux festes de Bacchus, que nous sçavons entre nous qui en [98 $\left.\mathrm{r}^{\circ}\right]$ sommes participans ${ }^{74}$. Doncques, presupposant l'ame estre immortelle ${ }^{75}$, imagine en toy qu'il luy avient de mesmes que és oyseaux qui sont pris : car, si l'ame se nourrit long temps avec le corps, et par grands maniements d'affaires et long usage s'accoustume et s'apprivoise en ceste vie, quand elle s'en deloge et s'en revole, elle y rentre tout à coup par le moyen des renaissances ${ }^{76}$, et ne cesse de s'empescher tousjours des passions et fortunes que nous avons icy. Et ne pense pas que la vieillesse soit tant maudite et blasmee sur ${ }^{77}$ les rides et le poil gris et la foiblesse du corps ; mais cest aage là a ce mal qui luy est plus à reprocher que nul autre ${ }^{78}$, qu'il esloigne l'ame et l'estrange du souvenir de ce qu'elle voyoit au lieu dont elle est venue ${ }^{79}$, et parmy les choses d'icy l'appesantit et la rend lourde et grossiere : car par les ans elle plie et contraint la forme et habitude de son estre, et garde et entretient celle qu'elle a prise, par le moyen de tant de choses qu'il luy a fallu endurer. Mais, au contraire, l'ame qui a demeuré peu de temps captive, aysément par les puissantes loix de sa nature est retenue en sa forme naturelle, n'ayant pris du corps [ $\left.98 \mathrm{v}^{\circ}\right]$ qu'une façon de ply encores fraiche et molle : car ainsi que le feu, si on l'esteint et incontinent après on le rallume, il flamboye et se reprent soudain; de mesme l'ame qui naguieres est partie de son origine, quand elle y reva bien tost, la reprent plus facilement, et ne pourroit avoir aucun avantage à reculer

de franchir au plus tost les portes de Pluton ${ }^{80}$, 
sinon pour nourrir en soy une grande amour des choses du monde : et, comme elle estoit charmee par le corps, s'amollir et destremper avecques luy.

[11] Et la verité de ceci se cognoit encores plus clair par les coustumes et loix anciennes de nostre cité : car, en nostre ville, on ne fait point de sacrifice à l'enterrement des enfants quand ils meurent, ny autre solennités ${ }^{\text {, }}$ comme il est raisonnable d'en faire pour les autres morts. Car les enfants ne tiennent rien de terrien ny des choses terrestres; et ne se dit point que leurs esprits, pour s'aymer pres de leurs corps, s'amusent et s'arrestent aux tombeaux et sepulchres, et aux repas qu'on a accoustumé presenter aux morts : car les loix ne souffrent point qu'on pense cela d'eux, comme [99 $\mathrm{r}^{\circ}$ ] n'estant point loysible de le croire de ceux là, desja estans en un estat meilleur et plus sainct, et au partir d'ici arrivez ${ }^{82}$ à une plus belle demeure ${ }^{83}$. Or, puis qu'à ne les en croire point, il y a plus de peine pour nous que de les en croire ${ }^{84}$, il faut par le dehors en user ainsi comme les loix l'ordonnent, et avoir le dedans encores moins souillé et mieux net, et plus chaste.

La fin en est à dire en Plutarque $e^{85}$.

\section{Lettre de Montaigne à sa femme (1570)}

Dédicace par Montaigne à son épouse Françoise de La Chassaigne de la Lettre de consolation de Plutarque à sa femme, traduite par La Boétie (notre texte 1), peu après la mort de leur premier enfant (fin août 1570). Cette lettre est la seule que nous ayons conservée de la correspondance de Montaigne avec son épouse.

Édition originale de référence : même édition que le texte 1, f. $89, \mathrm{r}^{\circ}-\mathrm{v}^{\circ}$.

Édition moderne : Montaigne, CEuvres complètes, éd. M. Rat, Paris, Gallimard «Bibliothèque de la Pléiade », 1962, p. 1371.

\section{A Mademoiselle ${ }^{86}$ de Montaigne, ma Femme}

Ma femme vous entendez bien que ce n'est pas le tour d'un galand homme, aux reigles de ce temps icy, de vous courtiser et caresser ${ }^{87}$ encore. Car ils disent ${ }^{88}$ qu'un habil homme peut bien prendre femme: mais que de l'épouser c'est à faire à un $\operatorname{sot}^{89}$. Laissons les dire : je me tiens de ma part à la simple façon du vieil aage, aussi en porteje tantost le poil ${ }^{90}$. Et de vray la nouvelleté couste si cher jusqu'à ceste heure à ce pauvre estat (et si je ne scay si nous en sommes à la derniere enchere ${ }^{91}$ ) qu'en tout et par tout j'en quitte le party ${ }^{92}$. Vivons ma femme, vous et moy, à la vielle Françoise. Or il vous peult souvenir comme feu Monsieur de la Boetie ce mien cher frere, et compaignon inviolable, me donna mourant ses papiers et ses livres, qui m'ont esté depuis le plus favory meuble des miens ${ }^{93}$. Je ne veulx pas chichement en user moy seul, ny ne merite qu'ils ne servent qu'à moy. A ceste cause il m'a pris envie d'en faire part à mes amis. Et par ce que je n'en ay, ce croy-je, nul plus privé que vous ${ }^{94}$, je vous envoye la Lettre consolatoire de Plutarque à sa femme, traduite par luy en François: bien 
marry dequoy la fortune vous a rendu ce present si propre, et que n'ayant enfant qu'une fille longuement attendue, au bout de quatre ans de nostre mariage, il a falu que vous l'ayez perdue dans le deuxiesme an de sa vie ${ }^{95}$. Mais je laisse à Plutarque la charge de vous consoler, et de vous advertir de vostre devoir en cela, vous priant le croire pour l'amour de moy: Car il vous descouvrira mes intentions, et ce qui se peut alleguer en cela beaucoup mieux que je ne ferois moymesmes.

Sur ce, ma femme, je me recommande bien fort à vostre bonne grâce, et prie Dieu qu'il vous maintienne en sa garde. De Paris, ce 10 septembre 1570.

Vostre bon mary,

Michel de Montaigne

\section{Louis-Ferdinand Céline, Voyage au bout de la nuit, extrait du chapitre 25 (1952)}

Édition de référence : Louis-Ferdinand Céline, Voyage au bout de la nuit, Paris, Gallimard, «NRF », 1952, p. 288-289.

Autre édition : Louis-Ferdinand Céline, Romans, Paris, Gallimard, « Bibliothèque de la Pléiade », 1981, t. I, p. 288-289.

Bardamu revient de l'institut médical Bioduret où il n'a pu se procurer un remède pour soigner le petit Bébert, sept ans, qu'il sait désormais condamné. Ne pouvant se résoudre à franchir la Seine vers Rancy, il s'attarde chez les bouquinistes et découvre notre texte 2. Céline en propose alors une récriture à sa façon, pleine d'ironie sans doute, mais pas dénuée pour autant d'une certaine tendresse.

[...] Les bouquinistes des quais fermaient leurs boîtes. « Tu viens ! » que criait la femme par-dessus le parapet à son mari, à mon côté, qui refermait lui ses instruments, et son pliant et les asticots. Il a grogné et tous les autres pêcheurs ont grogné après lui et on est remontés, moi aussi, là-haut, en grognant, avec les gens qui marchent. Je lui ai parlé à sa femme, comme ça pour lui dire quelque chose d'aimable avant que ça soye la nuit partout. Tout de suite, elle a voulu me vendre un livre. C'en était un de livre qu'elle avait oublié de rentrer dans sa boîte à ce qu'elle prétendait. « Alors ce serait pour moins cher, pour presque rien... » qu'elle ajoutait. Un vieux petit « Montaigne » un vrai de vrai pour un franc. Je voulais bien lui faire plaisir à cette femme pour si peu d'argent. Je l'ai pris son « Montaigne ${ }^{96}$ ».

Sous le pont, l'eau était devenue toute lourde. J'avais plus du tout envie d'avancer. Aux boulevards, j'ai bu un café crème et j'ai ouvert ce bouquin qu'elle m'avait vendu. En l'ouvrant, je suis juste tombé sur une page d'une lettre qu'il écrivait à sa femme le Montaigne, justement pour l'occasion d'un fils ${ }^{97}$ à eux qui venait de mourir. Ça m'intéressait immédiatement ce passage, probablement à cause des rapports que je faisais tout de suite avec Bébert. Ah ! qu'il lui disait le Montaigne, à peu près comme ça à son épouse. T'en fais pas va, ma chère femme! Il faut bien te consoler !... Ça s'arrangera !... Tout s'arrange dans la vie... Et puis d'ailleurs, qu'il lui disait encore,j'ai justement retrouvé 
hier dans des vieux papiers d'un ami à moi une certaine lettre que Plutarque envoyait lui aussi à sa femme dans des circonstances tout à fait pareilles aux nôtres... Et que je l'ai trouvée si joliment bien tapée sa lettre ma chère femme, que je te l'envoie sa lettre !... C'est une belle lettre! D'ailleurs je ne veux pas t'en priver plus longtemps, tu m'en diras des nouvelles pour ce qui est de guérir ton chagrin !... Ma chère épouse! Je te l'envoie la belle lettre! Elle est un peu là comme lettre celle de Plutarque !... On peut le dire! Elle a pas fini de t'intéresser !... Ah! non! Prenez-en connaissance ma chère femme! Lisez-la bien! Montrez-la aux amis. Et relisez-la encore! Je suis bien tranquille à présent! Je suis certain qu'elle va vous remettre d'aplomb!... Vostre bon mari. Michel. Voilà que je me dis moi, ce qu'on peut appeler du beau travail. Sa femme devait être fière d'avoir un bon mari qui s'en fasse pas comme son Michel. Enfin, c'était leur affaire à ces gens. On se trompe peut-être toujours quand il s'agit de juger le cœur des autres. Peut-être qu'ils avaient vraiment du chagrin ? Du chagrin de l'époque?

\section{NOTES}

1. Tanagra, ancienne cité grecque de Béotie.

2. En marge : Superfluité et vaine superstition à eviter.

3. Inconvenient : malheur.

4. Nourrir : élever, éduquer.

5. Et si sçais : tu sais aussi. (Conformément au texte grec de Plutarque.)

6. Il est traditionnel en Grèce de donner le nom du père à l'aîné des garçons, le nom de la mère à l'aînée des filles.

7. Dans le texte de Plutarque, c'est la fillette qui est «chérie tendrement ». La traduction de La Boétie suggère plutôt que Plutarque n'a qu'un nom féminin à aimer, celui que partagent son épouse et sa fille : Timoxéna.

8. En marge : Naturel gay d'une petite fille.

9. Du tout : complètement.

10. Attrempée : modérée.

11. La Boétie omet ici deux phrases de Plutarque : "Ce n'était pas seulement aux autres enfants, mais encore à ses joujoux favoris, à ses poupées, qu'elle voulait que sa nourrice donnât à téter. À ce sein, qui était comme sa table particulière, son humanité conviait tous ceux qui la rendaient heureuse : elle aimait à partager avec eux ce qu'elle avait de plus beau. » (trad. Ricard, 1844).

12. Travailler : faire souffrir.

13. En marge : Comment il se faut souvenir des morts.

14. Et s'applique à la lutte.

15. Le cormier est un bois souple dont on fait aisément des arcs. Plutarque disait simplement : « Je déteste mon arc et tous ces exercices. » Ces vers d'une tragédie d'Euripide, aujourd'hui perdue, Phaéton, nous ont été conservés par Plutarque. Voir Euripidis fragmenta, publiés par F.-G. Wagner, dans la Collection grecque de Didot, p. 806.

16. Montaigne semble se souvenir de ce passage dans Les Essais : «Plutarque mesme regrette sa fille par des singeries de son enfance» («De la diversion», III, 4, éd. P. Villey, Paris, PUF, «Quadrige ", 1965, p. 836). Toutes les références aux Essais renvoient à cette édition.

17. Un plaisir beaucoup plus grand que le chagrin.

18. En marge : Que le dueil doit estre moderé. 
19. Desconforter : désoler, chagriner.

20. De chagrins et de tourments.

21. N'en : ni en.

22. Quant à moi.

23. Prins : pris

24. En marge : La somptuosité ne servir de rien.

25. Esbahy: étonné.

26. En marge : Naturelle affection envers les morts.

27. Travaux : souffrances.

28. Du tout : complètement.

29. L'édition originale porte « force » (coquille que nous corrigeons).

30. Garder : empêcher.

31. En marge : Ancienne façon de porter le deuil.

32. Mal avenables: inconvenantes.

33. Faire compte de : attacher de l'importance à.

34. Hantez : fréquentés.

35. Accoustrement : habillement, sans valeur péjorative.

36. En marge : L'honneur que Plutarque fait à sa femme, et pourquoy il la louë tant.

37. Simplesse : modestie, humilité.

38. Noter la christianisation du texte.

39. L'aisné de nos enfants : prénommé Autobule.

40. Quand et eux : avec eux.

41. Que tout était en ordre.

42. À maint-un : à plus d'un, à de nombreuses personnes.

43. Tetin : sein.

44. Pour se servir d'eux, dirait-on, comme d'une distraction.

45. Indiscrettement : sans retenue.

46. Desconfort : chagrin.

47. Et qu'il soit ainsi : la preuve en est que.

48. En marge : Fable d'Esope touchant le dueil. Dans la Consolation à Apollonius, Plutarque donne une version moins allusive de cet apologue, «Jupiter et le Deuil»: «La reine Arsinoé [112a] était inconsolable de la mort de son fils; un ancien philosophe vint la trouver, et pour calmer sa douleur, usa de cet apologue : "Quand Jupiter distribua les emplois aux différents génies, le Deuil était absent. Le partage fait, il parut et demanda d'avoir son emploi comme les autres. Jupiter, qui les avait tous donnés, se trouva fort embarrassé, et n'ayant pas d'autre don à lui faire, il le chargea des honneurs qu'on rend aux morts, c'est-à-dire des regrets et des larmes. Ainsi, grande reine, comme les autres génies aiment ceux qui les honorent, de même le Deuil s'attache à ceux qui le servent. [112b] Si vous le méprisez, il s'éloignera de vous. Si, au contraire, vous lui rendez avec soin les honneurs auxquels il préside, c'est-à-dire les regrets et les larmes, il vous aimera et vous enverra sans cesse de quoi fournir son culte." Ce discours fit sur la reine la plus forte impression, et arrêta ses gémissements et ses plaintes. " Voir Aesopica, éd. Ben Edwin Perry, I, Greek and Latin Texts, Urbana (Illinois), Indiana University Press, 1952, fable $\mathrm{n}^{\circ} 462$. Cf. Camerarius, Aesopi Phrygis [...] Fabellae, Tübingen, U. Morhard, 1538, p. 296, «Luctus Praemia ». Pour des adaptations en vers français, $c f$. Philibert Guide, Les fables morales, éd. Laura Rovero, Genève - Paris, Slatkine - H. Champion, 1987, p. 38, fable VII ; et Pierre Defrasnay, Mythologie ou recueil des fables grecques, ésopiques et sybaritiques, Paris, 1750, p. 188 (livre XIII, fable XVII, « Jupiter le jour de sa fête... »).

49. Et non pas luy quitter le fort : et ne pas lui abandonner la place (métaphore militaire).

50. En laissant son habillement et son poil : en portant le deuil, en se rasant les cheveux. 
51. Tenir en serre : «Tenir en ses serres se dit proprement de quelqu'un de ces oiseaux [les oiseaux de proie] quand il tient entre ses griffes quelque petit oiseau; mais nostre langage use de cette phrase, parlant de celuy qui tient quelcun à sa mercy » (Henri Estienne, De la précellence du langage françois, éd. L. Feugère, Paris, Delalain, 1850, p. 133).

52. Entourné : entouré.

53. La traduction de La Boétie est ici lacunaire. On rétablit les mots manquants sur la base du texte grec.

54. Estuver: se laver, prendre un bain.

55. Failloit : fallait.

56. Ennuy : profond chagrin.

57. Ains : mais.

58. Il est très difficile de se reprendre, quand bien même on le voudrait.

59. En marge : Les visites de folles femmes à craindre.

60. Plutarque évoque cet ami dans plusieurs traités, notamment Pourquoi la Pythie ne rend plus ses oracles en vers, chap. VII. Dans le dialogue Sur l'E de Delphes (386d-387d), il est le porte-parole du point de vue stoïcien.

61. En marge : Exemples bien à propos.

62. En marge : Comment la consideration du temps peut servir de consolation.

63. Cet alinéa est le seul déjà présent dans l'édition de 1571. Il contribue à mettre en valeur cette maxime en marge : Qu'il se faut contenter de ce qui plait à Dieu. Plutarque évoquait pour sa part les Dieux.

64. En marge : Similitude à noter.

65. Sentiment : odorat.

66. Une peut-être, pas davantage.

67. Faire prendre grand saut : troubler profondément.

68. En marge : D'où depend la felicité.

69. Les «gens mecaniques" sont les ouvriers, mais il s'agit probablement d'une coquille pour « maniaques » (fous).

70. La traduction de La Boétie est ici lacunaire. On rétablit les mots manquants sur la base du texte grec.

71. Defaillent : manquent, font défaut.

72. En marge : Le lieu, où nous croyons les morts estre en repos, nous doit servir de consolation.

73. Aucuns tiennent : certains soutiennent.

74. Nous sommes mal renseignés sur ces mystères de Dionysos, du fait du silence imposé aux initiés. Mais Plutarque s'appuie sur les « sentences » apprises avec son épouse dans ce cadre pour démentir ceux qui professent l'opinion que les morts "n'ont en nul endroit nul mal ny tourment ». Dans le contexte chrétien qui est celui de La Boétie, la formule équivaut à refuser de nier l'existence de l'Enfer et accessoirement du Purgatoire.

75. En marge : L'ame estre immortelle.

76. C'est l'idée de métempsychose.

77. Sur : à cause de...

78. En marge : Quel mal à reprocher à la vieillesse.

79. Cette croyance rappelle la théorie platonicienne de la conception de l'âme dans le monde des idées.

80. Ce vers, dont Plutarque ne cite ici que la fin, est cité en entier dans la Consolation à Apollonius. Il est tiré d'une sentence célèbre de Théognis : «De tous les biens, le plus souhaitable pour les habitants de la terre, c'est de n'être point né, de n'avoir jamais vu les éclatants rayons du soleil ; ou bien, ayant pris naissance, de passer le plus tôt possible par la porte de Pluton, de reposer, profondément enseveli sous la terre. » (Sentences, v. 425-428).

81. En marge : Enterrement d'enfants sans solennité, et pourquoy. 
82. Au partir d'ici arrivez: arrivés en quittant ce monde.

83. La Boétie omet ou censure cette phrase de Plutarque : «Je n'ignore pas que sur ce point il y a lieu ici à bien des contestations. »Cette omission contribue à la christianisation du texte.

84. Autrement dit : « comme il est plus dangereux de se refuser à de semblables croyances que de les admettre »

85. Comprendre: «La fin manque dans l'édition de Plutarque ». C'est du moins ce qu'on croit au $\mathrm{XVI}^{\mathrm{e}}$ siècle puisque le texte ne présente pas de formule conclusive. Mais la critique actuelle pense au contraire que le texte est complet.

86. Mademoiselle : le mot désigne jusqu'au XVIII ${ }^{\mathrm{e}}$ siècle une femme mariée de la petite noblesse.

87. Caresser : flatter.

88. Ils disent : on dit (latinisme).

89. L'espouser: se lier à elle "par obligation mutuelle d'affection et d'estime» (A. J. Greimas). Même idée dans les Essais : « C'est trahison de se marier sans s'espouser » (III, 5, "Sur des vers de Virgile », p. 853).

90. Aussi je grisonne déjà.

91. Cf. Essais, III, 9, « De la vanité », p. 959 : « Nous ne sommes pas pourtant, à l'avanture, à nostre dernier periode ».

92. Allusion à la réforme protestante et à ses conséquences politiques en France : l'unité de l'État est gravement menacée et Montaigne craint à juste titre que les choses n'empirent encore. Cf. Essais, I, 23, "De la coutume et de ne changer aisément une loy receüe", p. 119: «Je suis desgousté de la nouvelleté [...]. »

93. Mon bien le plus précieux.

94. Montaigne hésitera dans les Essais sur les liens entre la relation maritale et l'amitié véritable : il les oppose dans « De l'amitié » (I, 28, p. 186) mais les rapproche dans « Sur des vers de Virgile » (III, 5, p. 851) et dans « De la vanité » (III, 9, p. 975).

95. En réalité, la première fille de Montaigne est morte à l'âge de deux mois, du moins si l'on en croit la mention manuscrite de l'auteur dans les Ephémérides de Beuther (f. $187 \mathrm{r}^{\circ}$ ) : « Junius 28. 1570. Naquit de Françoëse de la Chassaigne et de moë une fille que ma mère et mons ${ }^{r}$ le presidant de la Chassaigne père de ma fame surnomarent Thoinette. C'est le premier enfant de mon mariage, Et mourut deux moës après. » (Euvres complètes, op. cit., p. 1407, nº 13). Les critiques et éditeurs de cette lettre ont diversement interprété cette curieuse inexactitude. Maurice Rat semble y voir un simple lapsus: "Montaigne écrit ans pour mois" (éd.cit., p.1720, n. 2). Frédérique Leichter-Flack, dans un article sur Céline, emploie le terme de lapsus et juge que « ce lapsus peut être retenu contre Montaigne» («Intertextualité et exemplarité morale de la littérature : le cas de la souffrance de l'enfant, Dostoïevski / Platonov / Céline », dans E. Bouju, A. Gefen, G. Hautcœur et M. Macé dir., L'Exemplarité littéraire. Savoirs de l'exemple, pouvoirs de l'exemplaire, Rennes, PUR, 2007, p. 125).

Mais le plus récent biographe de Montaigne croit pour sa part à une véritable erreur de l'auteur sur l'âge de sa fille : « il ne manifeste pas une tendresse exagérée à l'égard des petits enfants. Il lui arrive même de se tromper sur le temps qu'a vécu leur première fille lorsqu'il écrit une lettre de consolation à sa femme. Il confond deux mois et deux ans ! (Ch. Bardyn, Montaigne, La splendeur de la liberté, Paris, Flammarion, 2015, p. 202). Cette interprétation est irrecevable pour plusieurs raisons : d'une part, on a vu que Montaigne note soigneusement sur son Ephemeris de Beuther les naissances, baptêmes, parrains et marraines de ses filles, mais aussi la date approximative de leur décès. D'autre part, la dédicace étant datée du 10 septembre, l'année même de la naissance et quelques jours après le décès de Thoinette, son premier et unique enfant à cette date, Montaigne ne saurait la croire morte à deux ans !

Plus séduisante sinon plus probable est l'hypothèse de P. J. Smith, qui voit là « sans doute une faute de l'imprimeur, qui ayant à l'esprit les deux ans de la fille de Plutarque a dû lire an au lieu de mois" (Réécrire la Renaissance, de Marcel Proust à Michel Tournier:exercices de lecture 
rapprochée, Amsterdam, Rodopi, 2009, p. 82). Toutefois cette hypothèse est fragile : d'une part les deux mots ne se ressemblent ni graphiquement, ni phonétiquement ; il paraît improbable de les confondre; d'autre part, cela suppose de la part du compositeur d'imprimerie une culture plutarquienne qu'il n'avait pas nécessairement (ce texte de Plutarque n'avait jamais été traduit en français auparavant).

Il nous semble qu'on peut formuler une autre hypothèse : Montaigne aurait bien écrit "an ", mais il ne s'agirait pas d'une erreur ; plutôt d'un arrangement de la vérité historique, d'une petite falsification si l'on veut. Comme l'a bien rappelé P. J. Smith, il ne s'agit pas ici d'une lettre privée mais d'une dédicace, dont la fonction est essentiellement rhétorique : il s'agit de mettre en valeur la lettre de Plutarque, de la recommander au lecteur. Quelle meilleure façon que de mettre en scène l'usage personnel que peuvent en faire Montaigne et sa femme pour se consoler euxmêmes? Pour persuader son lecteur de la valeur consolatoire de la lettre de Plutarque, Montaigne a donc tout intérêt à laisser croire qu'il a perdu un enfant déjà relativement avancé en âge (comme Plutarque et son épouse) plutôt qu'un nourrisson de deux mois (décès hélas banal, dont on se console probablement plus aisément à l'époque). Cette petite mystification renforce en outre l'identification implicite entre le couple Montaigne et le couple Plutarque, si l'on peut dire.

96. Nous ignorons de quelle édition du texte disposait Céline; il pourrait d'agir de l'édition de Montaigne en quatre tomes de la collection «Les meilleurs auteurs classiques français et étrangers » (Paris, Flammarion, 1908), où figure cette lettre (t. IV, p. 272) et que mentionne H. Godard dans son édition (op. cit., t. I, p. 1247).

97. On sait que Montaigne écrit en fait à l'occasion de la mort de sa première fille (il n'a eu que des filles). Erreur de Céline, qui a lu trop vite, ou plutôt liberté prise avec la vérité historique afin de faciliter le rapprochement de la situation de Bardamu avec celle de Montaigne, les « rapports » de cet imaginaire fils de Montaigne et de Bébert?

De même, on l'a vu, Montaigne jouait peut-être de l'âge de sa fille pour se rapprocher de Plutarque. 